\title{
Asymptotic Estimates of Stirling Numbers
}

\author{
By N. M. Temme
}

New asymptotic estimates are given of the Stirling numbers $S_{n}^{(m)}$ and $(S)_{n}^{(m)}$, of first and second kind, respectively, as $n$ tends to infinity. The approximations are uniformly valid with respect to the second parameter $m$.

\section{Introduction}

The Stirling numbers of the first and second kind, denoted by $S_{n}^{(m)}$ and $(3)_{n}^{(m)}$, respectively, are defined through the generating functions

$$
\begin{aligned}
& x(x-1) \cdots(x-n+1)=\sum_{m=0}^{n} S_{n}^{(m)} x^{m}, \\
& x^{n}=\sum_{m=0}^{n} \operatorname{SS}_{n}^{(m)} x(x-1) \cdots(x-m+1),
\end{aligned}
$$

where the left-hand side of (1.1) has the value 1 if $n=0$; similarly, for the factors in the right-hand side of (1.2) if $m=0$. This gives the 'boundary values'

$$
S_{n}^{(n)}=(S)_{n}^{(n)}=1, n \geq 0, \text { and } S_{n}^{(0)}=(S)_{n}^{(0)}=0, n \geq 1
$$

Furthermore, it is convenient to agree on $S_{n}^{(m)}=\left(S_{3}^{(m)}=0\right.$ if $m>n$.

The Stirling numbers are integers; apart from the above mentioned zero values, the numbers of the second kind are positive; those of the first kind have the sign of $(-1)^{n+m}$.

Address for correspondence: Professor N. M. Temme, CWI, P.O. Box 4079, 1009 AB Amsterdam, The Netherlands. e-mail: nicot@cwi.nl. 
Alternative generating functions are

$$
\begin{aligned}
& \frac{[\ln (x+1)]^{m}}{m !}=\sum_{n=m}^{\infty} S_{n}^{(m)} \frac{x^{n}}{n !}, \\
& \frac{\left(e^{x}-1\right)^{m}}{m !}=\sum_{n=m}^{\infty}\left(S_{n}^{(m)} \frac{x^{n}}{n !} .\right.
\end{aligned}
$$

The Stirling numbers play an important role in difference calculus, combinatorics, and probability theory. More properties of Stirling numbers can be found in, for example, Jordan [8, Chapter 4], Comtet [5], and in the chapter on combinatorial analysis in Abramowitz and Stegun [1, Chapter 24]. Recent interest in Stirling numbers can be found in Butzer et al. [3], [4].

Asymptotic expansions can be found in several papers. Hsu [7] has given an expansion for the numbers of the second kind that is useful when $n-m$ is small. Moser and Wyman [10], [11] have considered several overlapping domains in the $n, m$-plane with $n \geq m$. Bleick and Wang [2] have given a complete expansion for the numbers of the second kind, which is convergent when $m<(n+1)^{2 / 3} /\left[\pi+(n+1)^{-1 / 3}\right]$. When divergent, the expansion is still useful when used as an asymptotic series. However, the case $m \sim n$ (both large) is not covered. In Dingle [6, p. 199] the numbers of both kinds are considered. Again, the approximations are not uniformly valid with respect to $m$; the results for the numbers of the first kind become worse when $m \ll n$, whereas for the numbers of the second kind the problems arise when $m \sim n$. In most cases, the asymptotic results are obtained by using saddle point techniques for contour integrals. Recently, Knessl and Keller [9] have treated the asymptotics from a quite different point of view.

The purpose of the paper is to present new expansions for the Stirling numbers that hold uniformly with respect to $m$. The method is based on a modification of the saddle point method, as described in [13]. Short tables are given to show the results for $n=10$; further computer experiments confirm the uniform character of our estimates.

\section{Stirling numbers of the second kind}

An explicit representation of the numbers of the second kind is available as a finite sum:

$$
\left(3 \xi_{n}^{(m)}=\frac{1}{m !} \sum_{k=0}^{m}(-1)^{m-k}\left(\begin{array}{c}
m \\
k
\end{array}\right) k^{n} .\right.
$$

A proof follows by expanding the left-hand side of (1.4) with Newton's binomial formula and by comparing the power series of the resulting exponential functions with the right-hand side of (1.4). From (2.1) several limiting forms can be derived. However, more powerful results can be 
obtained from integral representations following from the generating functions. From (1.4) we obtain

$$
(3)_{n}^{(m)}=\frac{n !}{m !} \frac{1}{2 \pi i} \int \frac{\left(e^{x}-1\right)^{m}}{x^{n+1}} d x,
$$

where the contour of integration is a small circle around the origin. We write this in the form

$$
(3)_{n}^{(m)}=\frac{n !}{m !} \frac{1}{2 \pi i} \int e^{\phi(x)} \frac{d x}{x},
$$

where

$$
\phi(x)=-n \ln x+m \ln \left(e^{x}-1\right) .
$$

This integral will be estimated by using the saddle point method (for a general introduction to this topic see, for instance, Olver [12] or Wong [14]). The saddle point is defined by the solution of the equation $\phi^{\prime}(x)=0$. There is a real positive saddle point $x_{0}$ that solves the equation

$$
\frac{m}{n} x=1-e^{-x}
$$

The solution $x_{0}=0$ is not of interest since the contour in (2.3) is not allowed to pass through the origin. Moreover, $\phi^{\prime}(0)$ is undefined. When $m \ll n$, the solution $x_{0}$ approaches the origin, however, and when $m \sim n$, it approaches infinity. The saddle point method is based on replacing $\phi(x)$ by a quadratic function, for instance by writing $\phi(x)-\phi\left(x_{0}\right)=t^{2}$, a local transformation near $x=x_{0}$. However, straightforward application of the saddle point method gives approximations that are less accurate when $n \sim m$. To define a different transformation, we observe that when $x \rightarrow 0^{+}$, we have $\phi(x) \sim(m$ $-n) \ln x$, when $x \rightarrow \infty$, we have $\phi(x) \sim m x$. This suggests the transformation $x \rightarrow t(x)$ defined by

$$
\phi(x)=m t+(m-n) \ln t+A,
$$

where $A$ is not depending on $t$. The derivative of the right-hand side vanishes at $t_{0}=(n-m) / m$. We prescribe for the mapping in (2.5) the corresponding points

$$
x=0 \Leftrightarrow t=0, x=x_{0} \Leftrightarrow t=t_{0}, x=+\infty \Leftrightarrow t=+\infty .
$$

The quantity $A$ follows from substitution of $x=x_{0}, t=t_{0}$ in (2.6), which gives

$$
A=\phi\left(x_{0}\right)-m t_{0}+(n-m) \ln t_{0} .
$$


Transformation (2.6) brings (2.3) in the form

$$
\left(\xi_{n}^{(m)}=\frac{n !}{m !} \frac{e^{A}}{2 \pi i} \int e^{m t} f(t) \frac{d t}{t^{n-m+1}}\right.
$$

where

$$
f(t)=\frac{t}{x} \frac{d x}{d t}=\frac{m\left(t-t_{0}\right)}{x \phi^{\prime}(x)} .
$$

A transformation like (2.6) is investigated earlier in [13]. From the analysis of [13], it follows that the function $f$ is analytic in a neighborohood of the origin, and in a wider domain of the complex plane, including the positive real axis. Especially, $f$ is analytic at $t=0$ and at $t=t_{0}$, also when $t_{0}$ (that is, $x_{0}$ ) tends to zero.

Initially, the contour in (2.7) is a small circle around $t=0$, but it can be deformed into a contour through the new saddle point $t_{0}$. A first approximation to $(3)_{n}^{(m)}$ is now obtained by replacing $f(t)$ in (2.7) with the value of this function at the saddle point $t_{0}$. The remaining integral is easily evaluated, giving the one-term approximation

$$
(3)_{n}^{(m)} \sim e^{A} m^{n-m} f\left(t_{0}\right)\left(\begin{array}{c}
n \\
m
\end{array}\right), \text { as } n \rightarrow \infty
$$

where

$$
f\left(t_{0}\right)=\frac{1}{x_{0}} \sqrt{\frac{m t_{0}}{\phi^{\prime \prime}\left(x_{0}\right)}}=\sqrt{\frac{t_{0}}{\left(1+t_{0}\right)\left(x_{0}-t_{0}\right)}} .
$$

The value of $f\left(t_{0}\right)$ follows from applying l'Hôpital's rule on the second form in (2.8), giving

$$
f\left(t_{0}\right)=\frac{m}{x_{0} \phi^{\prime \prime}\left(x_{0}\right) d x / d t}
$$

Using this in comparison with the first form in (2.8), we obtain an expression of $d x / d t$ evaluated at $x=x_{0}, t=t_{0}$. This finally gives $f\left(t_{0}\right)$. In section 4 a second term in the asymptotic estimate (2.9) is given.

We compute the limiting values of $f\left(t_{0}\right)$. Let $\nu=m / n$, and consider $\nu \rightarrow 1$. It easily follows that $x_{0}=2(1-\nu)+\mathscr{O}\left[(1-\nu)^{2}\right]$. Hence,

$$
f\left(t_{0}\right)=1+\mathscr{O}(1-\nu), \text { as } \nu \rightarrow 1 \text {. }
$$

When $\nu \rightarrow 0$, we have $x_{0} \sim 1 / \nu$, giving

$$
f\left(t_{0}\right)=1+\mathscr{O}(\nu) \text {, as } \nu \rightarrow 0
$$


In Table 1 we give the exact values of $\left(S_{10}^{(m)}(m=1,2, \ldots, 10)\right.$ and the approximations based on (2.9) (the value for $m=10$ is not computed via (2.9)). The maximal relative error is 0.0064 , and occurs at $m=3$. Similar computations with $n=20, n=30$ show the following: the maximal errors are $0.0031,0.0021$, and occur for $m=7, m=10$, respectively. Observe that the maximal errors do not occur at boundary values of $m$, but at about $m=\frac{1}{3} n$. Larger values of $n$ also show the uniform character of the asymptotic estimate (2.9).

\section{Stirling numbers of the first kind}

Dingle [6] has used the generating function (1.3) giving the representation

$$
S_{n}^{(m)}=\frac{n !}{m !} \frac{1}{2 \pi i} \int \frac{[\ln (z+1)]^{m}}{z^{n+1}} d z
$$

where the contour is a small circle around $z=0$. To make the integral representation similar to the previous case, he transformed $1+z=e^{x}$. This gives

$$
S_{n}^{(m)}=\frac{n !}{m !} \frac{1}{2 \pi i} \int \frac{x^{m} e^{x}}{\left(e^{x}-1\right)^{n+1}} d x
$$

Integrating by parts and $x \rightarrow-x$ gives

$$
S_{n}^{(m)}=\frac{(n-1) !}{(m-1) !} \frac{(-1)^{n-m}}{2 \pi i} \int \frac{x^{m-1}}{\left(1-e^{-x}\right)^{n}} d x
$$

Again, the contour is a small circle around $x=0$. This representation has been used by Dingle to apply the saddle point method. We have used the method of the previous section on this integral, but the results were quite inaccurate.

Table 1

Approximations of the Stirling numbers of the second kind

\begin{tabular}{cccccc}
\hline$m$ & $(3)_{10}^{(m)}$ & $(2.9)$ & $m$ & $\left(S_{10}^{(\mathrm{m})}\right.$ & $(2.9)$ \\
\hline 1 & 1 & 0.9993 & 6 & 22827 & 22922 \\
2 & 511 & 512.98 & 7 & 5880 & 5897.7 \\
3 & 9330 & 9390.1 & 8 & 750 & 751.45 \\
4 & 34105 & 34319 & 9 & 45 & 45.04 \\
5 & 42525 & 42750 & 10 & 1 & 1 \\
\hline
\end{tabular}


To try an alternative representation, we turn to (1.1). It is easy to verify that

$$
(-1)^{n-m} S_{n+1}^{(m+1)}=\frac{1}{2 \pi i} \int \frac{(x+1)(x+2) \cdots(x+n)}{x^{m+1}} d x=\frac{1}{2 \pi i} \int e^{\phi(x)} \frac{d x}{x},
$$

where

$$
\phi(x)=\ln [(x+1)(x+2) \cdots(x+n)]-m \ln x
$$

The saddle point is the solution of $\phi^{\prime}(x)=0$. When $1 \leq m \leq n-1$, there is one positive solution $x_{0}$. To prove this, note that $\phi^{\prime}(x)$ is negative when $x$ is small and that for positive values of $x$ we have $\phi^{\prime}(x)>n /(x+n)-m / x$. Hence, $\phi^{\prime}(x)>0$ when $x>n m /(n-m)$. This shows that $\phi^{\prime}$ has at least one positive zero. Next we observe that $\phi^{\prime}$ has at least one zero in any of the $n-1$ intervals $(-2,-1),(-3,-2), \ldots,(-n,-n+1)$. Now we reduce the $n+1$ fractions of $\phi^{\prime}$ to a common denominator. Then the numerator of $\phi^{\prime}$ is a polynomial having degree $n$ and having at most $n$ zeros. From the distribution of the zeros of $\phi^{\prime}$ just mentioned, we infer that $\phi^{\prime}$ indeed has one and only one positive zero. axis:

The present function $\phi(x)$ has the following behavior on the positive real

$$
\phi(x) \sim-m \ln x \text {, as } x \rightarrow 0, \phi(x) \sim(n-m) \ln x \text {, as } x \rightarrow \infty .
$$

Combining these two limiting cases, we observe that the function $n \ln (x+1)$ $-m \ln x$ has (globally on $(0, \infty))$ the same graph as $\phi(x)$. This suggests the following transformation $x \rightarrow t(x)$

$$
\phi(x)=n \ln (1+t)-m \ln t+B .
$$

The derivative of the right-hand side vanishes at $t_{0}=m /(n-m)$. We prescribe for the mapping in (3.2) the corresponding points

$$
x=0 \Leftrightarrow t=0, x=x_{0} \Leftrightarrow t=t_{0}, x=+\infty \Leftrightarrow t=+\infty .
$$

The quantity $B$ follows from substitution of $x=x_{0}, t=t_{0}$ in (3.2), which gives

$$
B=\phi\left(x_{0}\right)-n \ln \left(t_{0}+1\right)+m \ln t_{0} .
$$

Transformation (3.2) brings (3.1) in the form

$$
(-1)^{n-m} S_{n+1}^{(m+1)}=\frac{e^{B}}{2 \pi i} \int \frac{(1+t)^{n}}{t^{m+1}} g(t) d t
$$


where, initially, the contour is a small circle around $t=0$, and

$$
g(t)=\frac{t}{x} \frac{d x}{d t}=\frac{(n-m) t-m}{(t+1) x \phi^{\prime}(x)} .
$$

A first approximation to $S_{n+1}^{(m+1)}$ is now obtained by replacing $g(t)$ in (3.3) with the value of this function at the saddle point $t_{0}$. The remaining integral is evaluated by using

$$
\frac{1}{2 \pi i} \int \frac{(1+t)^{n}}{t^{m+1}} d t=\left(\begin{array}{c}
n \\
m
\end{array}\right)
$$

This gives the one-term approximation

$$
S_{n+1}^{(m+1)} \sim(-1)^{n-m} e^{B} g\left(t_{0}\right)\left(\begin{array}{c}
n \\
m
\end{array}\right) \text {, as } n \rightarrow x \text {. }
$$

We finally compute $g\left(t_{0}\right)$. It follows from (3.4) that

$$
g\left(t_{0}\right)=\frac{t_{0}}{x_{0}} \frac{d x}{d t}=\frac{n-m}{\left(t_{0}+1\right) x_{0} \phi^{\prime \prime}\left(x_{0}\right) d x / d t},
$$

where $d x / d t$ is evaluated at $t=t_{0}$. This gives a relation for $d x / d t$ at $t=t_{0}$, from which we obtain

$$
g\left(t_{0}\right)=\frac{1}{x_{0}} \sqrt{\frac{m(n-m)}{n \phi^{\prime \prime}\left(x_{0}\right)}} .
$$

The function $\phi(x)$ and its derivatives occurring in the above formulas are elementary functions, and can be computed straightforwardly. However, when $n$ and $x$ are large, one may use representations in terms of the logarithm of the gamma function and the derivatives thereof. That is

$$
\begin{aligned}
\phi(x) & =\ln \Gamma(x+n+1)-\ln \Gamma(x+1)-m \ln x \\
\phi^{\prime}(x) & =\psi(x+n+1)-\psi(x+1)-m / x
\end{aligned}
$$

Asymptotic expansions of these functions are given in [1, chapter 6].

The mapping defined in (3.2) is of the same kind as the one in (2.6). The analytic properties again follow from the results in [13].

Remark: A curious finite expansion follows by substituting

$$
g(t)=\sum_{k=0}^{\infty} c_{k} t^{k}
$$


in (3.3), from which we obtain the exact representation

$$
(-1)^{n-m} S_{n+1}^{(m+1)}=e^{B} \sum_{k=0}^{m} c_{k}\left(\begin{array}{c}
n \\
m-k
\end{array}\right) .
$$

For example, when $m=0$, we have $c_{0}=g(0)=1, e^{B}=n !$, giving $S_{n+1}^{(1)}=$ $(-1)^{n} n !$.

In Table 2 exact values of $\left|S_{10}^{(m)}\right|,(m=1, \ldots, 10)$ are compared with absolute values of the approximations given in (3.5) (the values for $m=1$, $m=n$ are not computed via (3.5)). The maximal relative error now occurs at $m=3$, and is 0.0082 . For $n=20, n=30$, the maximal errors are: 0.0063 and 0.0053 , respectively; again they occur at $m=3$. These experiments confirm the uniform character with respect to $m$ of the result in (3.5).

\section{Higher order approximations}

The estimates in (2.9) and (3.5) can be supplied with more terms, and eventually written as complete asymptotic expansions. We concentrate on the numbers of the second kind; the treatment for the numbers of the first kind is more complicated owing to the special form of the function $\phi$ in (3.2).

Consider the integral in (2.7) in the form

$$
F_{\lambda}(m)=\frac{1}{2 \pi i} \int e^{m t} f(t) \frac{d t}{t^{\lambda+1}}
$$

where $m$ and $\lambda$ are positive integers. The contour is a small circle around the origin, where $f$ should be analytic (the method also works (with minor modifications) when $m$ and $\lambda$ are complex numbers). Writing

$$
f(t)=f(\mu)+(t-\mu) g(t), \mu=\lambda / m,
$$

we obtain

$$
F_{\lambda}(m)=\frac{m^{\lambda}}{\Gamma(\lambda+1)} f(\mu)+\frac{1}{2 \pi i} \int(t-\mu) e^{m t} g(t) \frac{d t}{t^{\lambda+1}} .
$$

Table 2

Approximations of the Stirling numbers of the first kind

\begin{tabular}{cccccc}
\hline$m$ & $\left|S_{10}^{(m)}\right|$ & $(3.5)$ & $m$ & $\left|S_{10}^{(m)}\right|$ & $(3.5)$ \\
\hline 1 & 362880 & 362880 & 6 & 63273 & 63007 \\
2 & 1026576 & 1018563 & 7 & 9450 & 9420.8 \\
3 & 1172700 & 1163168 & 8 & 870 & 868.2 \\
4 & 723680 & 718718 & 9 & 45 & 44.95 \\
5 & 269325 & 267855 & 10 & 1 & 1 \\
\hline
\end{tabular}




\section{Writing}

$$
\frac{1}{2 \pi i} \int(t-\mu) g(t) e^{m t-\lambda \ln t} \frac{d t}{t}=\frac{1}{2 m \pi i} \int g(t) d e^{m t-\lambda \ln t}
$$

and integrating by parts, we obtain

$$
F_{\lambda}(m)=\frac{m^{\lambda}}{\Gamma(\lambda+1)} f(\mu)-\frac{1}{2 m \pi i} \int f_{1}(t) e^{m t} \frac{d t}{t^{\lambda+1}},
$$

where

$$
f_{1}(t)=t \frac{d}{d t} g(t)=t \frac{d}{d t} \frac{f(t)-f(\mu)}{t-\mu} .
$$

Repeating this procedure (observe that the final integral has the same form as the starting function (4.1)), we can obtain eventually

$$
F_{\lambda}(m) \sim \frac{m^{\lambda}}{\Gamma(\lambda+1)} \sum_{k=0}^{\infty}(-1)^{k} f_{k}(\mu) m^{-k}
$$

where the functions $f_{k}(t)$ are defined by

$$
f_{k+1}(t)=t \frac{d}{d t} \frac{f_{k}(t)-f_{k}(\mu)}{t-\mu}, k=0,1,2, \ldots,
$$

with $f_{0}(t)=f(t)$. This procedure gives for (2.9) a complete asymptotic expansion of the form

$$
\operatorname{BSS}_{n}^{(m)} \sim e^{A} m^{n-m}\left(\begin{array}{c}
n \\
m
\end{array}\right) \sum_{k=0}^{\infty}(-1)^{k} f_{k}\left(t_{0}\right) m^{-k} \text {, as } n \rightarrow \infty,
$$

where $t_{0}=\mu=(n-m) / m$ and $f$ is given by (2.8). The first coefficient of the series is given by $(2.10)$, the second one, $f_{1}\left(t_{0}\right)$, can be obtained as follows. The function $f$ is analytic in a domain containing the positive real axis. Consider the Maclaurin expansion around $t=t_{0}$ (the point where we expect the main contributions to the integral in (2.7))

$$
f(t)=\sum_{k=0}^{\infty} a_{k}\left(t-t_{0}\right)^{k}, a_{k}=\frac{1}{k !} f^{(k)}\left(t_{0}\right) .
$$

Then it follows that

$$
f_{1}(t)=t \sum_{k=0}^{\infty}(k+1) a_{k+2}\left(t-t_{0}\right)^{k}
$$


and that $f_{1}\left(t_{0}\right)=t_{0} a_{2}$. Further coefficients $f_{k}\left(t_{0}\right)$ can also be expressed in terms of $a_{k}$, the derivatives of $f$ at the point $t_{0}$.

To compute $a_{k}$, we need the coefficients $x_{k}$ in the expansion

$$
x=x_{0}+x_{1}\left(t-t_{0}\right)+x_{2}\left(t-t_{0}\right)^{2}+\cdots,
$$

where $x_{0}$ is the solution of (2.5) and $x_{1}=a_{0} x_{0} / t_{0}=f\left(t_{0}\right) x_{0} / t_{0}$. See the first relation in (2.8); $f\left(t_{0}\right)$ is given in (2.10). With (2.8) we can express other values $a_{k}$ in terms of $x_{k}$. So we obtain by formal manipulations of power series

$$
\begin{aligned}
& a_{1}=\frac{2 t_{0} x_{0} x_{2}+x_{1} x_{0}-t_{0} x_{1}^{2}}{x_{0}^{2}} \\
& a_{2}=\frac{3 x_{3} t_{0} x_{0}+2 x_{2} x_{0}^{2}-3 t_{0} x_{0} x_{1} x_{2}-x_{1}^{2} x_{0}+t_{0} x_{1}^{3}}{x_{0}^{3}} .
\end{aligned}
$$

Hence, for $f_{1}\left(t_{0}\right)=t_{0} a_{2}$ we need $x_{0}, x_{1}, x_{2}, x_{3}$. To obtain $x_{2}, x_{3}$, we substitute (4.3) in the second relation of (2.8). After several manipulations we finally obtain the coefficient of the second term in (4.2), that is

$$
f_{1}\left(t_{0}\right)=\frac{\begin{array}{r}
-2 x_{0}^{3}+2 t_{0}^{5}+4 t_{0}^{3}+4 t_{0}^{4}+3 x_{0}^{2} t_{0}-6 x_{0} t_{0}^{4}-5 x_{0}^{2} t_{0}^{2} \\
+2 x_{0}^{4} t_{0}+x_{0}^{3} t_{0}-6 x_{0}^{3} t_{0}^{2}+8 x_{0}^{2} t_{0}^{3}
\end{array}}{24 a_{0}\left(1+t_{0}\right)^{2}\left(x_{0}-t_{0}\right)^{4}}
$$

A further analysis shows that $f_{1}\left(t_{0}\right)$ is a bounded function of $t_{0}$ on $[0, \infty)$. Using the two-term variant of (2.9), that is,

$$
\left(\xi_{n}^{(m)} \sim e^{A} m^{n-m}\left(\begin{array}{c}
n \\
m
\end{array}\right)\left[f\left(t_{0}\right)-\frac{f_{1}\left(t_{0}\right)}{m}\right] \text {, as } n \rightarrow \infty,\right.
$$

we obtain the following maximal relative errors:

$$
\begin{aligned}
& n=10: \text { relative error }=0.00047 \text { occurs at } m=4 \\
& n=20: \text { relative error }=0.00012 \text { occurs at } m=3 \\
& n=30: \text { relative error }=0.00006 \text { occurs at } m=4 \\
& n=40: \text { relative error }=0.00003 \text { occurs at } m=5 \\
& n=50: \text { relative error }=0.00002 \text { occurs at } m=7
\end{aligned}
$$

\section{References}

1. M. Abramowitz and I. A. Stegun, Handbook of Mathematical Functions with Formulas, Graphs and Mathematical Tables, National Bureau of Standards Applied Series 55, U.S. Government Printing Office, Washington, D.C., 1964. 
2. W. E. Bleick and P. C. C. Wang, Asymptotics of the Stirling numbers of the second kind, Proc. Amer. Math. Soc. 42:575-580 (1974).

3. P. L. Butzer and M. Hauss, On Stirling functions of the second kind, Stud. Appl. Math. 84:71-91 (1991).

4. P. L. Butzer, M. Hauss, and M. Schmidr, Factorial functions and Stirling numbers, Results in Mathematics 16:16-33 (1989).

5. L. COMTET, Advanced Combinatorics, D. Reidel, Dordrecht-Holland, 1974.

6. R. B. Dingle, Asymptotic Expansions: Their Derivation and Interpretation, Academic Press, New York, 1973.

7. L. C. Hsu, Note on an asymptotic expansion of the $n$-th difference of zero, Annals of Mathematical Statistics 19:273-277 (1948).

8. C. Jordan, The Calculus of Finite Differences, Chelsea Publishing Company, New York, 1947.

9. C. H. KNEssl and J. B. Keller, Stirling number asymptotics from recursion equations using the ray method, Stud. Appl. Math. 84:43-56 (1991).

10. L. MOSER and M. Wyman, Asymptotic development of the Stirling numbers of the first kind, J. London Math. Soc. 33:133-146 (1958).

11. L. MOSER and M. WYMAN, Stirling numbers of the second kind, Duke Math. J. 25:29-43 (1958).

12. F. W. J. Olver, Asymptotics and Special Functions, Academic Press, New York, 1974.

13. N. M. TEMmE, Laplace integrals: Transformation to standard form and uniform asymptotic expansion, Quart. Appl. Math. XLIII:103-123 (1985).

14. R. Wong, Asymptotic Approximations of Integrals, Academic Press, New York, 1989.

\section{CENTRUM VOOR WiSKUNDE EN INFORMATICA}

(Received February 18, 1992) 